\title{
Precipitation Characteristics and Mechanism of Vanadium Carbides in a V-Microalloyed Medium-Carbon Steel
}

\author{
Xiao-Lin Pan ${ }^{1} \cdot$ Minoru Umemoto ${ }^{2}$ \\ Received: 25 March 2018/Revised: 22 April 2018/Published online: 29 June 2018 \\ (C) The Chinese Society for Metals and Springer-Verlag GmbH Germany, part of Springer Nature 2018
}

\begin{abstract}
The precipitation characteristics and mechanism of vanadium carbides during isothermal transformation at $650{ }^{\circ} \mathrm{C}$ in a V-microalloyed medium-carbon steel were investigated through scanning electron microscopy and transmission electron microscopy as well as dilatometry test. Five morphologies of vanadium carbides were found to precipitate at different nucleation sites during the transformation. Two kinds of interphase precipitation form simultaneously in both pro-eutectoid and pearlitic ferrites. The linear arrays of fine interphase precipitates are parallel to the $\gamma / \alpha$ interface, and the fine needles of interphase precipitates are perpendicular to the $\gamma / \alpha$ interface. The vanadium carbides of long or short fibers, coarse particles and fine particles form in both pro-eutectoid and pearlitic ferrites, displaying different precipitation distributions and orientation relationships with ferrite. The precipitation mechanisms of vanadium carbide precipitates with different modes were proposed, and the precipitation sequence of various vanadium carbide precipitates was finally ascertained.
\end{abstract}

Keywords Microalloyed steels · Carbide precipitation · Isothermal transformation · Precipitation sequence · Orientation relationship

\section{Introduction}

The precipitation modes of vanadium carbide (VC), especially for interphase precipitation in low-carbon V-microalloyed steels, have been widely investigated [1-4]. Interphase precipitation with irregularly spaced and curved sheets of VC particles usually occurs at high transformation temperatures above $800{ }^{\circ} \mathrm{C}$, then interphase precipitation with regularly spaced and planar sheets of VC particles takes place as the temperature decreases, and finally, matrix precipitation from supersaturated ferrite becomes dominant when the temperature decreases from $700{ }^{\circ} \mathrm{C}$ [5]. However, the fibrous precipitation in low-carbon HSLA steels, especially in pearlitic ferrite, was paid relatively less attention than interphase precipitation.

Available online at http://link.springer.com/journal/40195

Xiao-Lin Pan

panxl@smm.neu.edu.cn

1 School of Metallurgy, Northeastern University, Shenyang 110819, China

2 Department of Mechanical Engineering, Toyohashi University of Technology, Toyohashi 441-8580, Japan
Edmonds found that [6] the volume fraction of fibrous VC is about $5 \%$ in an $\mathrm{Fe}-0.2 \mathrm{C}-1.0 \mathrm{~V}$ steel, but it increases to $20 \%$ when $1.5 \% \mathrm{Mn}$ or $1.5 \% \mathrm{Ni}$ is added. Moreover, Barbacki and Honeycombe reported that [7] lowering the transformation temperature can change the VC morphology from interphase to fibrous. Todd and $\mathrm{Li}$ studied the relationship between the interphase precipitation reactions and the mechanical properties in an $\mathrm{Fe}-0.2 \mathrm{C}-1.0 \mathrm{~V}-0.5 \mathrm{Mn}$ steel and found that the greatest contribution to precipitation strengthening is due to the interphase and matrix precipitation of VC particles, but the fibrous VC precipitates could not be associated with low values of the Charpy impact toughness [8].

For medium- and high-carbon steels, the coexistence of pro-eutectoid ferrite and pearlite results in more complicated morphologies of $\mathrm{VC}$ precipitates. Khalid and Edmonds observed that parallel $\mathrm{VC}$ precipitates form in an $\mathrm{Fe}-0.82 \mathrm{C}-11.90 \mathrm{Mn}-0.48 \mathrm{~V}$ steel, which is almost perpendicular to the interface in pearlitic ferrite [9]. Six classifications of $\mathrm{VC}$ morphologies were found to form in lowcarbon steels [8], but whether they can precipitate in medium-carbon steels or not is still unclear. Meanwhile, for fibrous precipitates, Khalid et al. and Smith et al. considered them as interphase precipitates $[9,10]$, but 

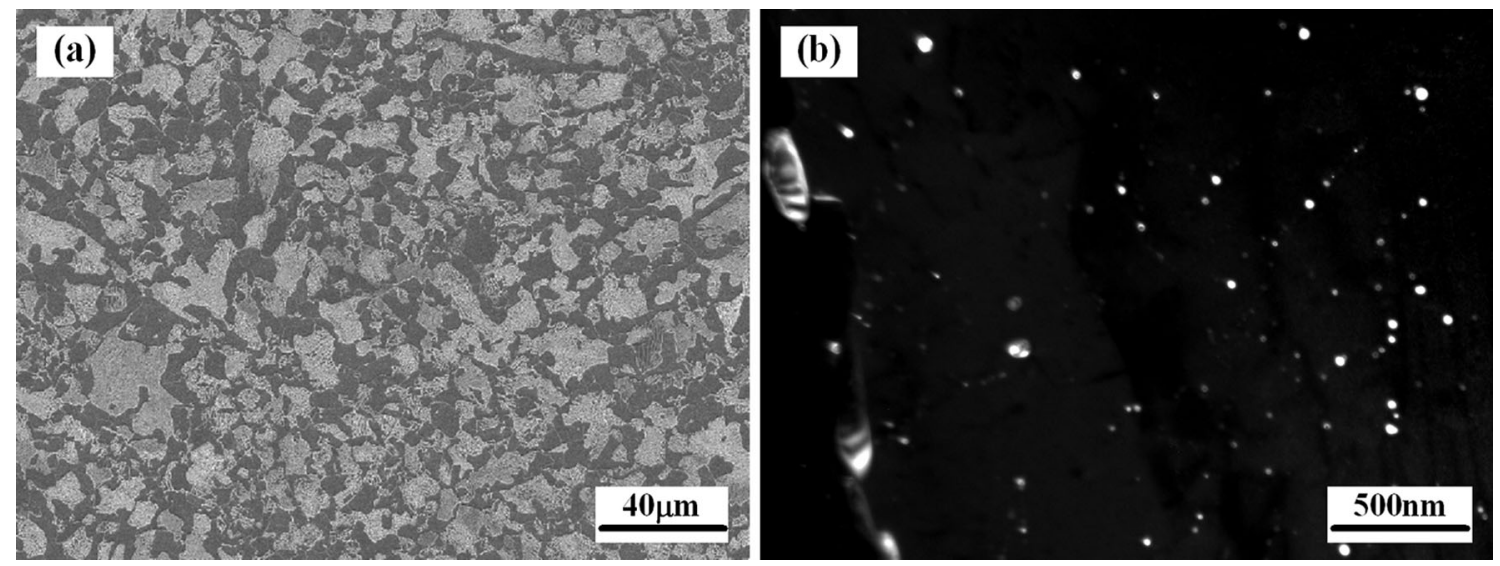

Fig. 1 Microstructures of the as-received steel: a SEM micrograph of pro-eutectoid ferrite and pearlite; $\mathbf{b}$ dark-field TEM micrograph of VC precipitates in pro-eutectoid ferrite

Todd et al. regarded them as coarsening of VC particles along dislocations [8]. The differences between them are still unrevealed. Furthermore, the precipitation sequence of various morphologies of $\mathrm{VC}$ precipitates is also unknown.

It is known that the V-microalloyed medium-carbon steels have been increasingly used in the automotive industry due to the improved tensile and impact properties $[11,12]$. The improvement in the mechanical properties has been mainly attributed to the precipitation of $\mathrm{VC}$ in both pro-eutectoid ferrite and pearlitic ferrite. In order to improve the mechanical properties of medium-carbon $\mathrm{V}$-microalloyed steels, it is of great importance to control the morphology, size and distribution of VC precipitates. The purpose of this research was to study the various kinds of VC precipitation in both pro-eutectoid ferrite and pearlitic ferrite after isothermal transformation below $\mathrm{A}_{1}$ temperature. Meanwhile, the mechanisms of different modes of $\mathrm{VC}$ precipitation and the precipitation sequence were also discussed.

\section{Experimental}

The chemical composition of the steel used in this investigation was $\mathrm{Fe}-0.47 \mathrm{C}-0.25 \mathrm{Si}-0.87 \mathrm{Mn}-0.30 \mathrm{~V}$ (wt $\%$ ). The ingot was produced via vacuum induction melting and then fabricated to 25 -mm-diameter rods by two-step extrusion and finally isothermally held at $850{ }^{\circ} \mathrm{C}$ for $15 \mathrm{~min}$ followed by air cooling to room temperature.

Cylindrical specimens of $10 \mathrm{~mm}$ long with diameter of $3 \mathrm{~mm}$ were machined. A 2-mm hole in diameter was drilled from one side of the specimen center to accommodate a thermocouple. The specimens were first induction-heated to the austenitization temperature of $1200{ }^{\circ} \mathrm{C}$ for $10 \mathrm{~min}$ using a Formaster-EDP machine in vacuum condition, cooled to $650{ }^{\circ} \mathrm{C}$ at a cooling rate of $50{ }^{\circ} \mathrm{C} / \mathrm{s}$, held for $100 \mathrm{~s}$ and $1 \mathrm{~h}$, respectively, and then quickly quenched to room temperature. Meanwhile, the dilatometry results were also synchronously determined during the above isothermal transformation.

The specimens after transformation were ground, polished and etched in a 5\% nital scanning electron microscope (SEM, JEOL JSM-6300) observation. Thin foils for transmission electron microscopy (TEM) examination were cut from the top of specimens and ground to disks with 50 $\mu \mathrm{m}$ in thickness. The disks were then electropolished in a Struers Tenupol-5 jet polisher using an electrolyte of $10 \%$ perchloric acid by volume in ethanol at $-20{ }^{\circ} \mathrm{C}$ and $20 \mathrm{~V}$. The TEM tests were carried out using a JEM-2100F microscope operating at $200 \mathrm{kV}$.

\section{Results}

Figure 1a shows the microstructure of the as-received steel consisting of the pro-eutectoid ferrite and pearlite. The VC precipitates were observed to exist in both pro-eutectoid ferrite and pearlitic ferrite through the TEM examinations, and the particle size ranges from 10 to $60 \mathrm{~nm}$. Most of the precipitates are randomly dispersed, as shown in Fig. 1b, though some precipitates are aligned in rows, which are associated with interphase precipitation.

The dilatometry results during the isothermal transformation as shown in Fig. 2 demonstrate that the austenite decomposition starts at $40 \mathrm{~s}$ and finishes at $2100 \mathrm{~s}$ when held at $650{ }^{\circ} \mathrm{C}$. The VC precipitation was observed obviously from the slope of the dilatometry curve during the transformation. The isothermally transformed microstructure after austenitization is shown in Fig. 3a. The grains grow to about $500 \mu \mathrm{m}$ during the austenitization process. The pearlites exhibit varieties of morphologies as shown in Fig. 3b, including classical lamellar pearlite and 


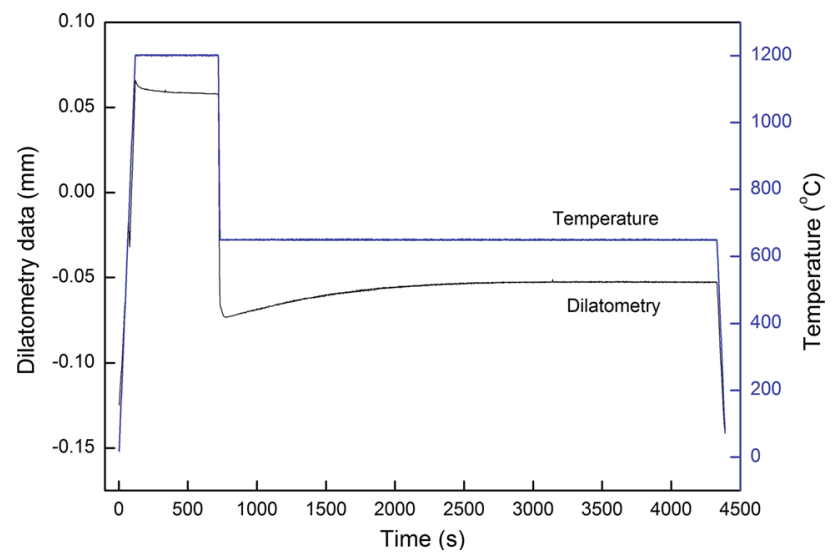

Fig. 2 Dilatometry results of the steel during the isothermal transformation

discontinuous lamellar pearlite. The pro-eutectoid ferrites are prior to form along the austenite grain boundaries. Short precipitates less than $200 \mathrm{~nm}$ were observed by SEM within the pro-eutectoid ferrite, as presented in Fig. 3c. Meanwhile, long precipitates up to $1 \mu \mathrm{m}$ in length are also present next to the ferrite grain boundaries as shown in Fig. 3d.
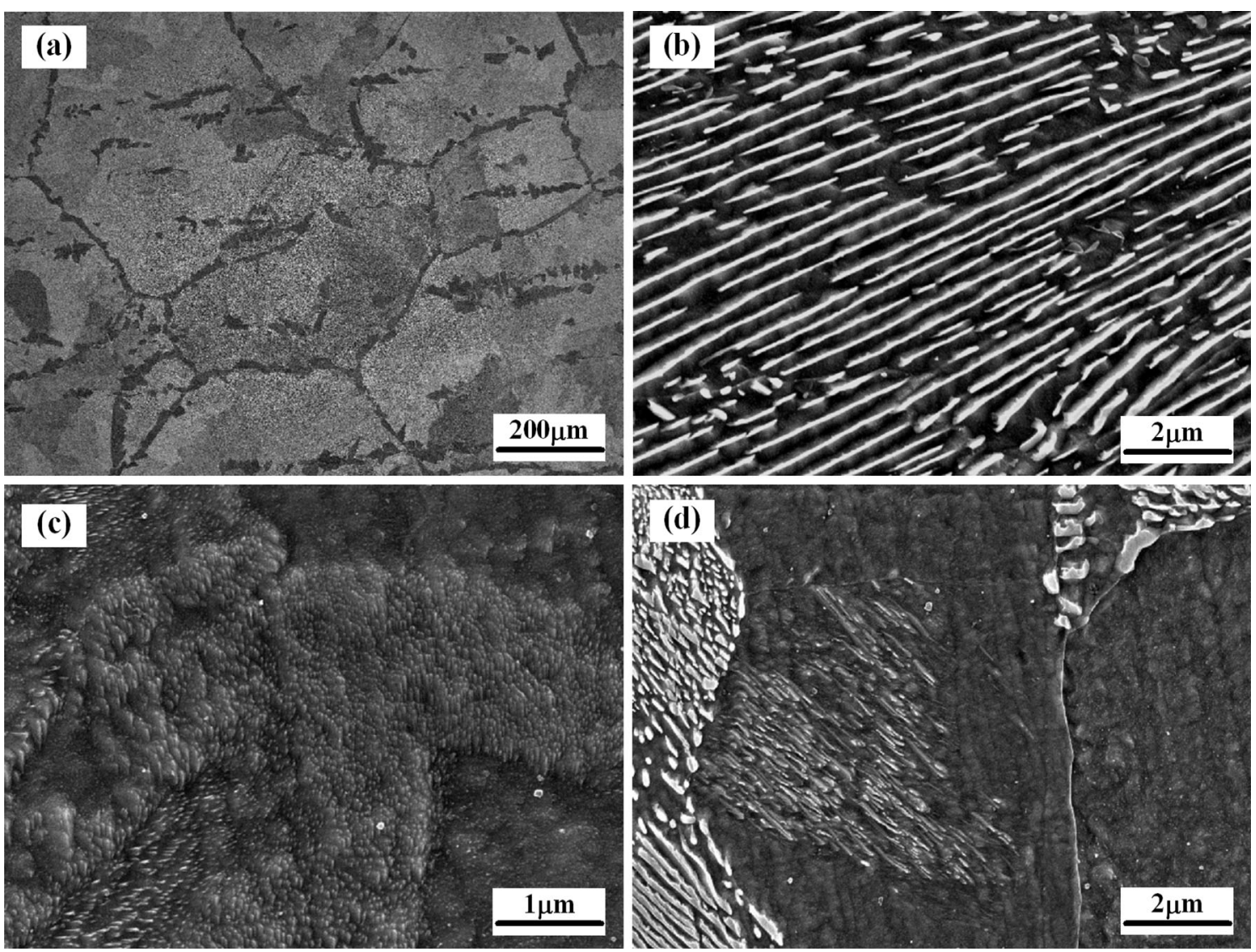

Fig. 3 SEM micrographs after isothermal transformation at $650{ }^{\circ} \mathrm{C}$ for $1 \mathrm{~h}$ : a low magnification; b typical pearlite morphology; c, d short and long precipitates of VC in pro-eutectoid ferrite 

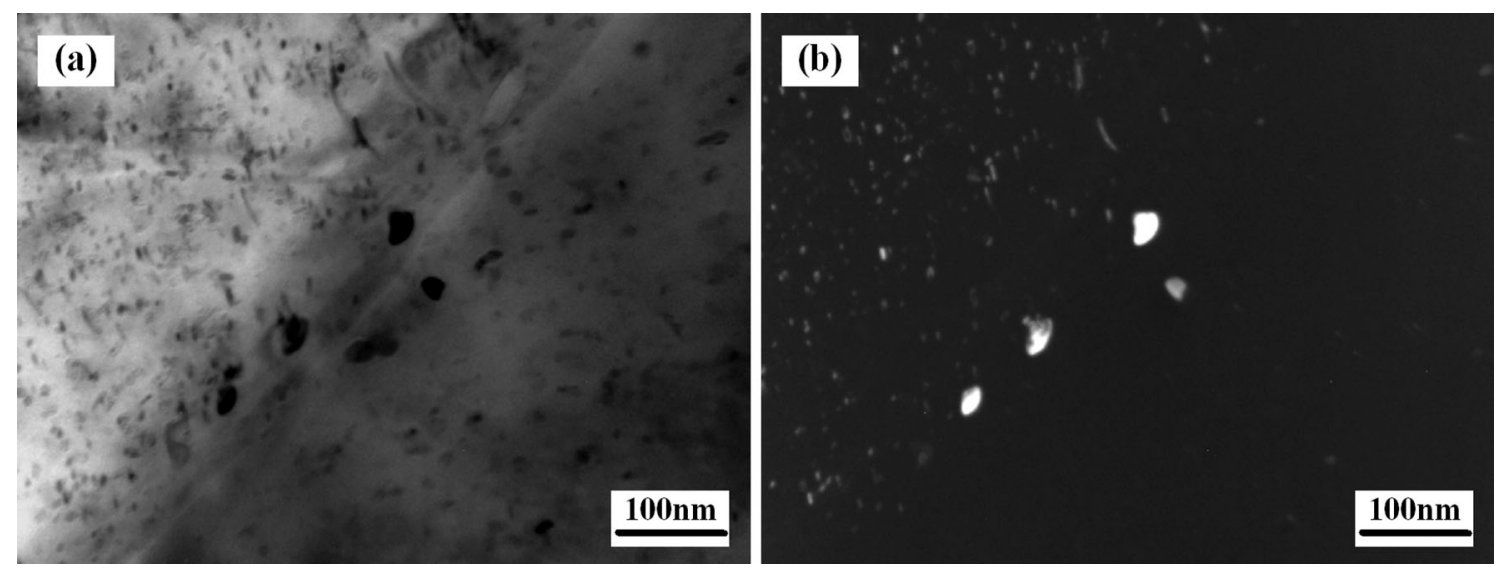

Fig. 4 TEM micrographs of coarse VC precipitation in austenite along the ferrite grain boundaries (holding for $1 \mathrm{~h}$ ): $\mathbf{a}$ bright-field image; $\mathbf{b}$ darkfield image
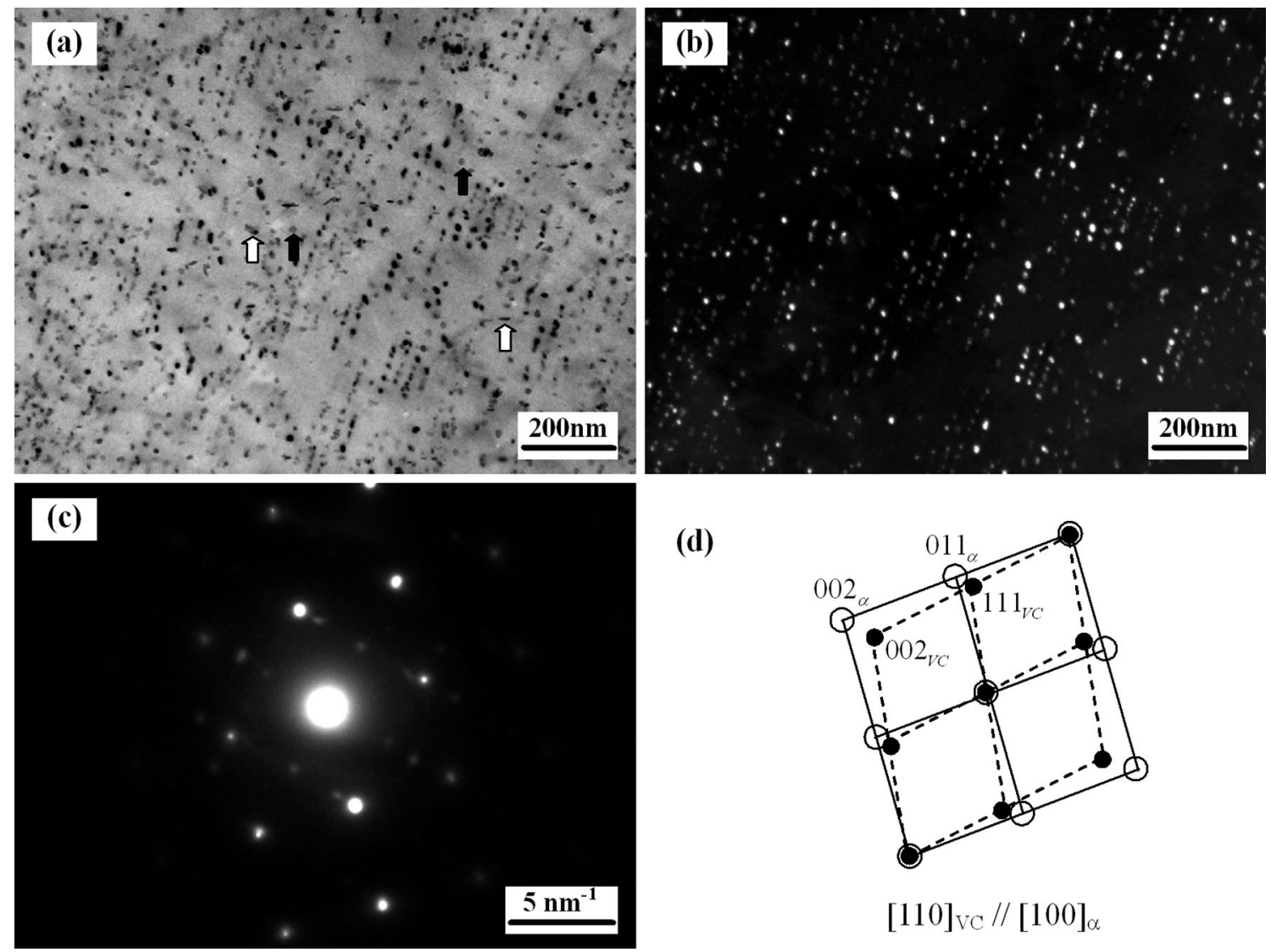

(d)

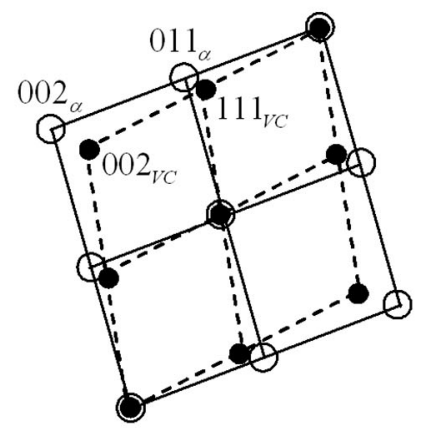

$$
[110]_{\mathrm{vC}} / /[100]_{\alpha}
$$

Fig. 5 TEM micrographs of planar interphase precipitation in pro-eutectoid ferrite (holding for $1 \mathrm{~h}$ ): a bright-field image; $\mathbf{b}$ dark-field image; c SAED pattern; $\mathbf{d}$ corresponding analysis

\subsection{VC Precipitation in Pro-eutectoid Ferrite}

Figure 5a, b shows the typical morphology of linear arrays of fine VC particles formed by the planar interphase precipitation in pro-eutectoid ferrite. The fine-banded VC particles are formed in sheets parallel to the $\gamma / \alpha$ interface as the transformation front moves through the austenite. The SAED pattern analyses in Fig. $5 c$, d indicate that these particles obey one of the three variants of the $\mathrm{BN}$ orientation relationship with ferrite. The intersheet spaces were determined to be $30-35 \mathrm{~nm}$. Moreover, the VC particles display different sizes of smaller particles (about $2 \mathrm{~nm}$ ) and 

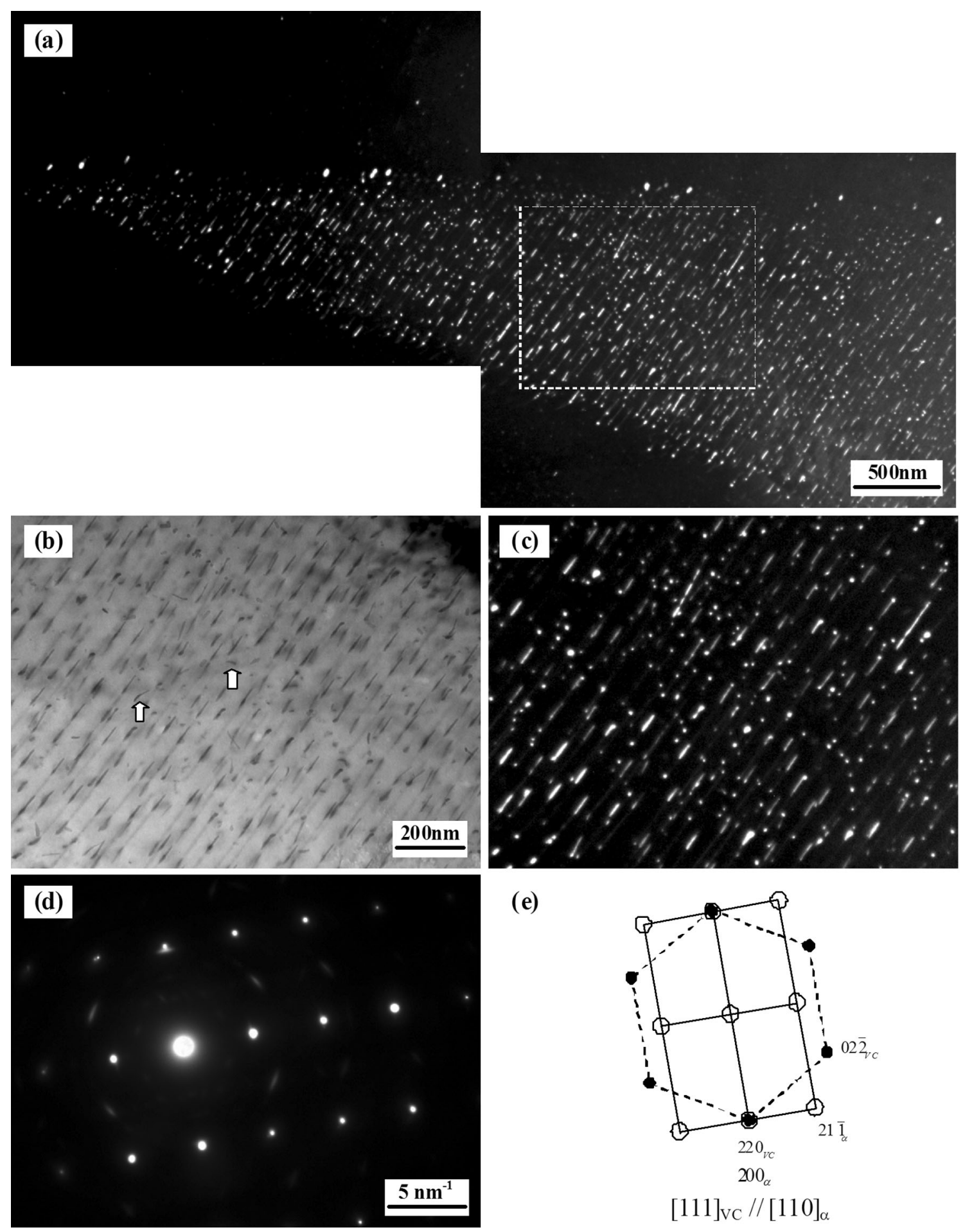

(e)

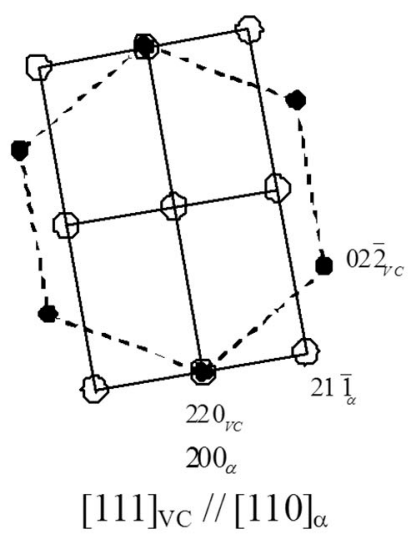

Fig. 6 TEM micrographs of fine needles of interphase precipitates in pro-eutectoid ferrite (holding for $1 \mathrm{~h}$ ): a dark-field image; $\mathbf{b}$, $\mathbf{c}$ bright-field and dark-field images of higher magnification of rectangular area in a; d SAED pattern; e corresponding analysis

larger particles (about $10 \mathrm{~nm}$ ). The larger particles may coarsen along the dislocations during the isothermal transformation.

Figure 6 shows another morphology of VC precipitates with $50-100 \mathrm{~nm}$ in length forming by interphase precipitation, which is also observed in Fig. 3c by SEM. The fine needles of VC precipitates, referred to "fibrous" $\mathrm{VC}$ in some references $[7,9,10]$, also form in sheets perpendicularly to the $\gamma / \alpha$ interface (Fig. 6a). These precipitates named "needlelike" VC in this study are normally 

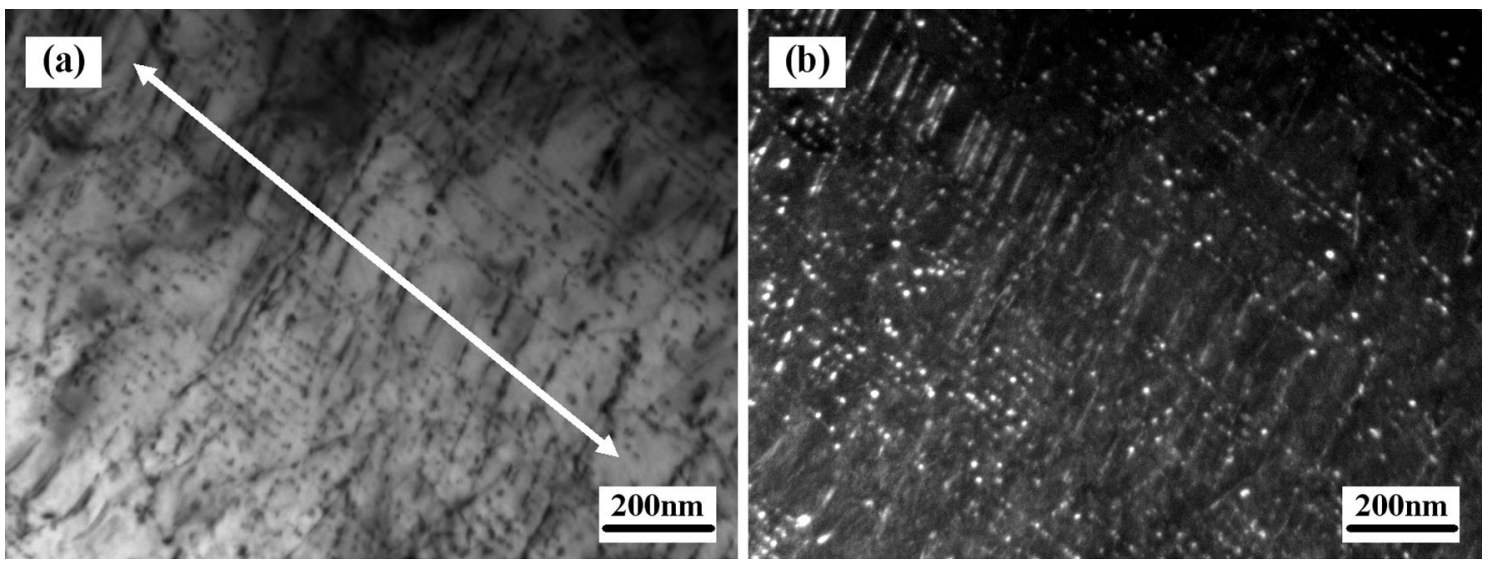

Fig. 7 TEM micrographs of co-precipitation of banded interphase precipitates and needlelike interphase precipitates in pro-eutectoid ferrite (holding for $1 \mathrm{~h}$ ): a bright-field image; b dark-field image
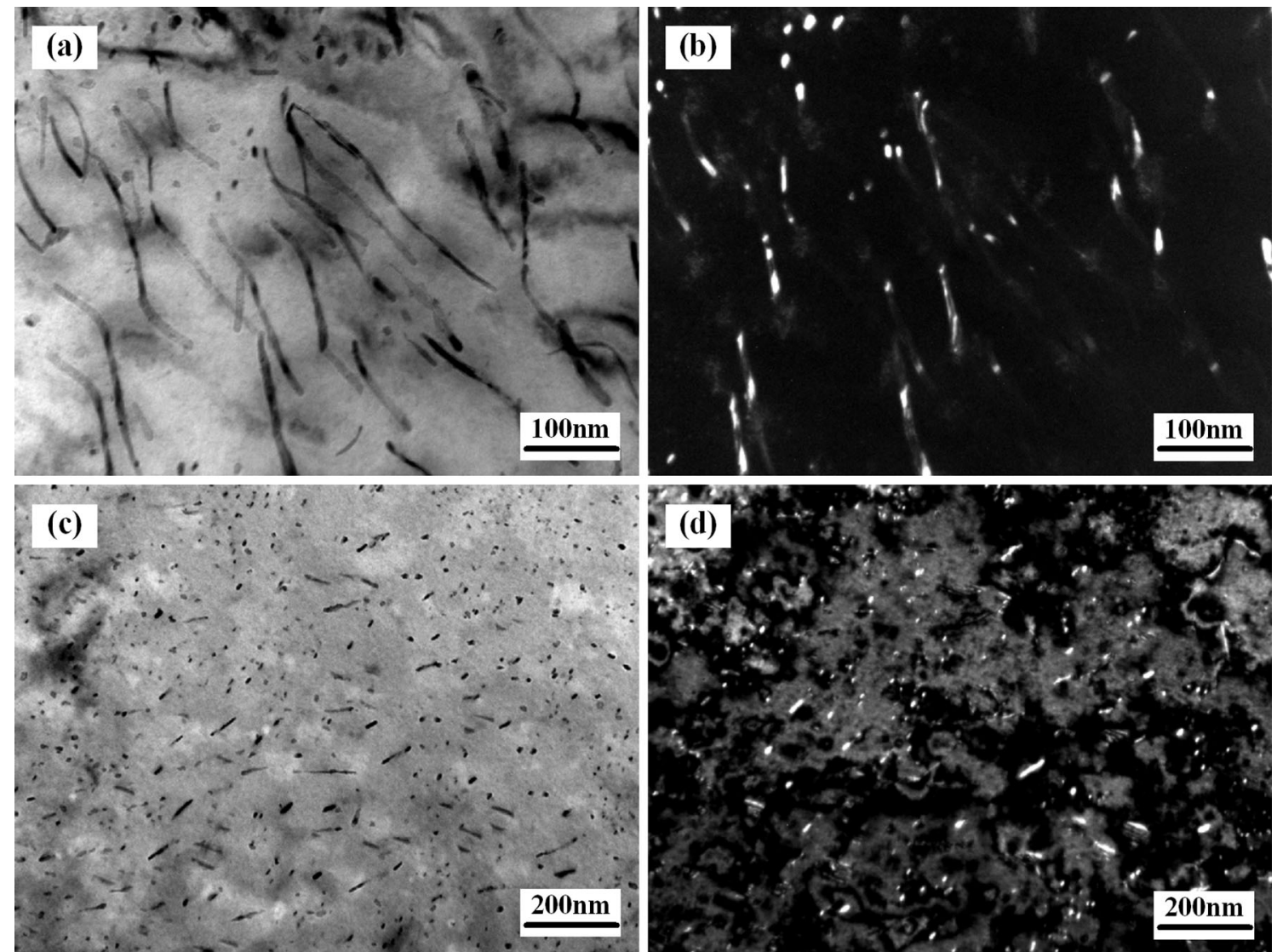

Fig. 8 TEM micrographs of fibrous VC precipitation in pro-eutectoid ferrite (holding for $1 \mathrm{~h}$ ): $\mathbf{a}$, $\mathbf{c}$ bright-field image; $\mathbf{b}, \mathbf{d}$ dark-field image

straight without any dendrite. They exhibit a single variant of the BN orientation relationship, as illustrated in Fig. 6d, e. The intersheet spaces of needlelike $\mathrm{VC}$ ranging from 50 to $200 \mathrm{~nm}$ are much larger than those of banded VC. Meanwhile, the banded interphase precipitates and the needlelike interphase precipitates can exist in one grain of ferrite, sometimes even in the same region, as observed in Fig. 7. The banded precipitates and the needlelike precipitates are almost perpendicular to each other. The morphologies of interphase precipitates suddenly change from fine banded to parallel needlelike in a limited region (see the arrow in Fig. 7a) during the isothermal transformation. The SAED pattern analyses show that the two different morphologies of interphase precipitates obey the same BN orientation relationship with ferrite. 

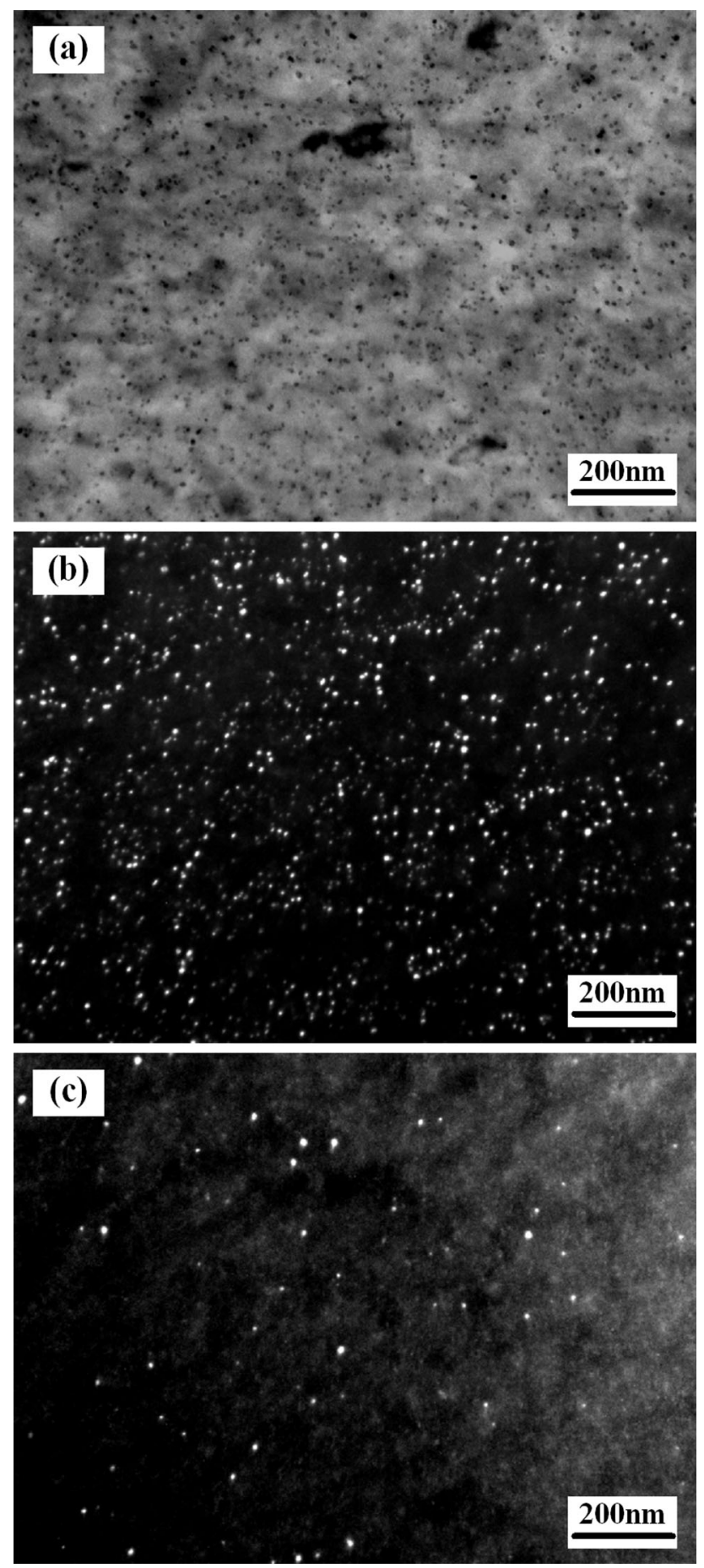

Fig. 9 TEM micrographs of matrix precipitation in pro-eutectoid ferrite (holding for $1 \mathrm{~h}$ ): a bright-field image; b, c dark-field image showing different orientations

Long fibers of VC precipitates were found to form near the ferrite grain boundaries as well as the transitional regions between pro-eutectoid ferrite and pearlite, as shown in Figs. 8a, b and 3d. These fibers, which are absolutely different from the needlelike VC precipitates, are usually curved and randomly distributed with longer than $200 \mathrm{~nm}$ in length, and some fibers are even as long as $1 \mu \mathrm{m}$. Short fibers of VC precipitates with approximately $10-100 \mathrm{~nm}$ in length as shown in Fig. 8c, d can occur almost everywhere in pro-eutectoid ferrite, even in the regions where the interphase precipitation takes place (shown in Figs. 5a and 6b by white arrows). The long and short fibers usually exhibit multiple variants of the BN orientation relationship, which sometimes show a single variant at a local region.

Figure 9a shows the morphology of fine VC particles formed by matrix precipitation, which randomly distribute in pro-eutectoid ferrite in different orientations as presented in Fig. 9b, c. The VC particles are very fine $(2-10 \mathrm{~nm})$, which precipitate in multiple variants of the BN orientation relationship. Because the first-formed ferrite grows too fast to form interphase precipitates, the ferrite becomes supersaturated so that the matrix precipitation takes place subsequently. The randomly distributed particles can also be found in the regions where the interphase precipitation occurs (see Fig. 5a by black arrows and Fig. 6c).

\subsection{VC Precipitation in Pearlite}

All morphologies of VC precipitates above in pro-eutectoid ferrite can be found in pearlite. The bands of fine VC particles formed by planar interphase precipitation often precipitate within the ferrite of discontinuous lamellar pearlite, as shown in Fig. 10a, b. The axis of the linear arrays is almost normal to the cementite long axis, which is consistent with the results of previous studies [15]. Occasionally, fine needles of VC precipitates formed by needlelike interphase precipitation were observed in pearlitic ferrite, as shown in Fig. 10c, d. The needlelike precipitates are parallel to each other and incline at an angle to the cementite long axis, which was also found by Khalid and Edmonds [8]. The SAED pattern analyses demonstrate that both finely banded and needlelike VC precipitates display a single variant of the $\mathrm{BN}$ orientation relationship with respect to the pearlitic ferrite.

Figure 10e, f shows long fibers (long arrows in Fig. 10f) and short fibers (short arrows in Fig. 10f) of VC particles precipitating in pearlite with spheroidized cementite. In classical lamellar pearlite, fine VC particles usually precipitate randomly, as shown in Fig. 10g, h, exhibiting all the three variants of the BN orientation relationship. In fact, the short fibrous and the randomly distributed particles precipitate all over the pearlitic ferrite, even in the regions where the interphase precipitation occurs (arrows in Fig. 10a, c), which are the same as those in pro-eutectoid ferrite. 

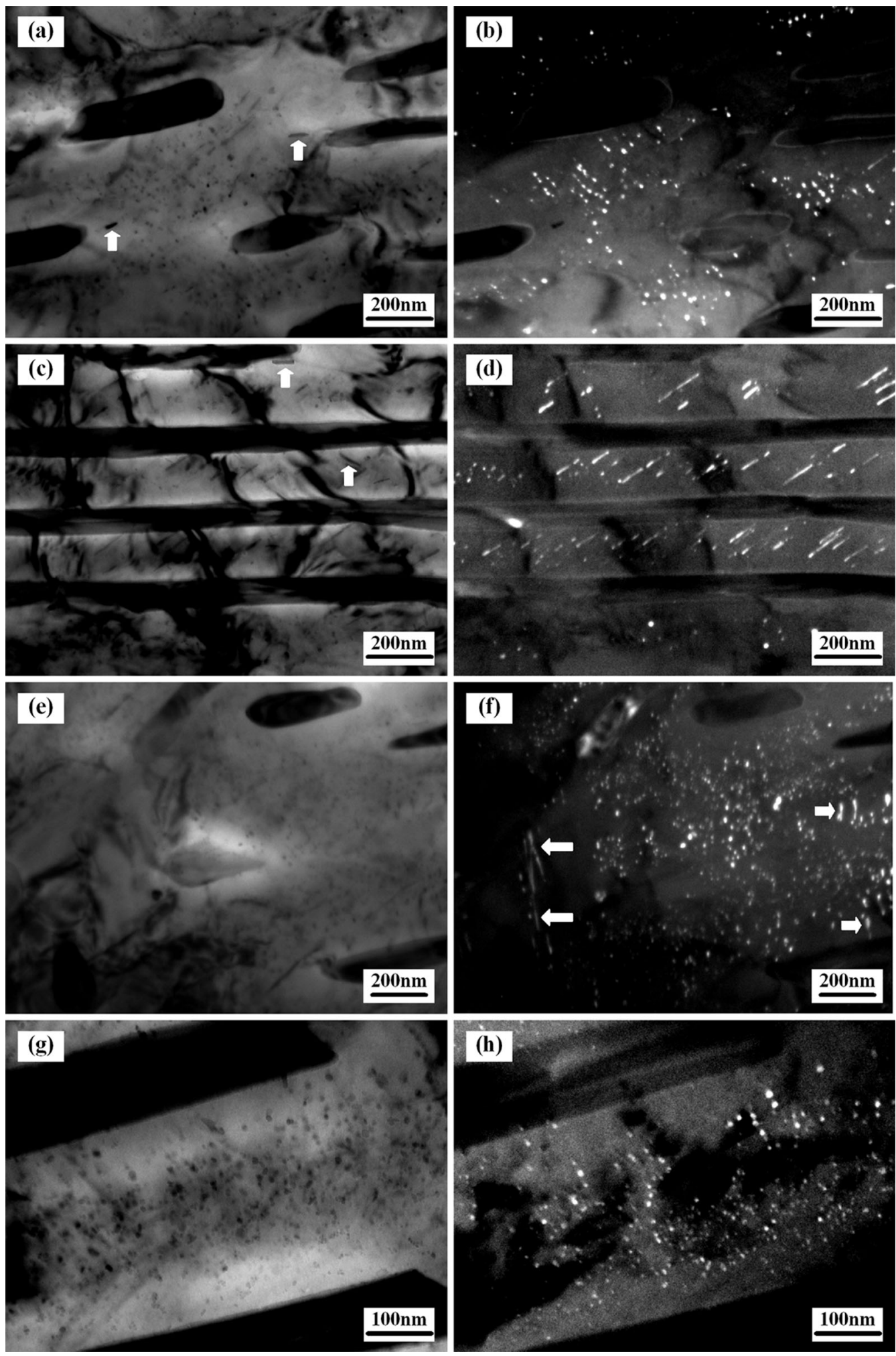

Fig. 10 TEM micrographs of VC precipitates in pearlite (holding for $1 \mathrm{~h}$ ): $\mathbf{a}, \mathbf{c}, \mathbf{e}, \mathbf{g}$ bright-field image; $\mathbf{b}, \mathbf{d}, \mathbf{f}, \mathbf{h}$ dark-field image 


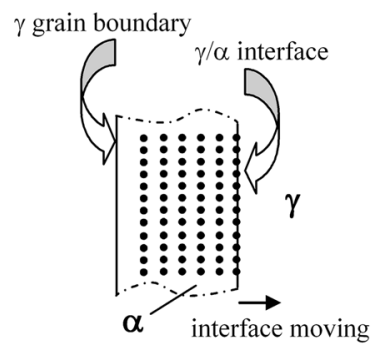

(a)

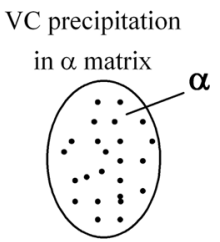

(c)

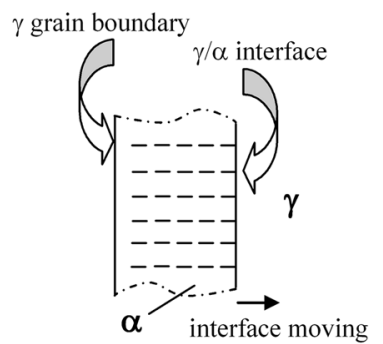

(b)

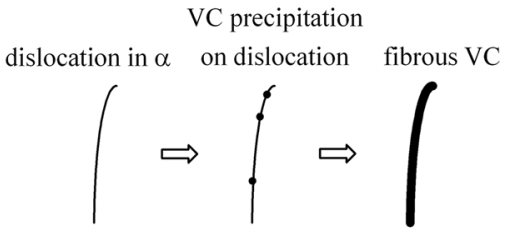

(d)
Fig. 11 Schematic diagrams of VC precipitation: a planar interphase precipitation; b needlelike interphase precipitation; c matrix precipitation; d fibrous precipitation

\section{Discussion}

\subsection{Mechanism for VC Precipitation}

The mechanisms or models of interphase precipitation were proposed by many researchers, which can be divided into two categories: one is the interface boundary energy controlled mechanism, such as the ledge mechanism [16], the bowing mechanism [17], the quasi-ledge mechanism [18] and the eutectoid decomposition model [19]; the other is the diffusion-controlled mechanism, such as the solute-drag nucleation model [6] and the solute balance model [20].

According to the solute-drag nucleation model, the buildup of carbon ahead of the moving austenite-ferrite boundary as well as the possible collection of vanadium atoms at the interface exerts a drag on its movement and
Table 1 Precipitation sequence of various morphologies of VC precipitates

\begin{tabular}{lll}
\hline Sequence & Morphology & Nucleation site \\
\hline 1 & Coarse particles & $\gamma /$ grain boundary \\
2 & Fine particles on planar sheets & $\gamma / \alpha$ interface \\
3 & Fine needles on planar sheets & $\gamma / \alpha$ interface \\
4 & Long or short fibers & Dislocations in $\alpha$ \\
5 & Fine particles & $\alpha$ matrix \\
\hline
\end{tabular}

slows the boundary migration sufficiently to allow nucleation and growth of $\mathrm{VC}$ precipitates [6]. As the $\mathrm{VC}$ growth continues, the carbon and vanadium at the interface are consumed, and the driving force increases sufficiently to move the transformation front to a position where nucleation is repeated, leading to the occurrence of planar interphase precipitation parallel to the $\gamma / \alpha$ interface (Fig. 11a). If the VC precipitates are oriented with a major growth direction parallel to the movement of the interface and the interfacial movement is slow enough, needlelike interphase precipitation perpendicular to the $\gamma / \alpha$ interface will occur (Fig. 11b). However, if the interface moves too fast, the interphase precipitation cannot happen, which results in the supersaturated ferrite. Then, matrix precipitation with a random distribution in ferrite will take place subsequently (Fig. 11c).

At the same time, dislocations are generated during the $\gamma / \alpha$ transformation due to the stress buildup with only small volume changes. Furthermore, the dislocation density increases with decreasing transformation temperature. Figure 12a shows that dislocations are generated during the transformation, especially near the ferrite grain boundaries. As the dislocations can provide preferential sites for nucleation, the VC particles nucleate on the dislocations as observed in Fig. 12b. Subsequently, these particles grow along the dislocations to form the fibrous morphology. The
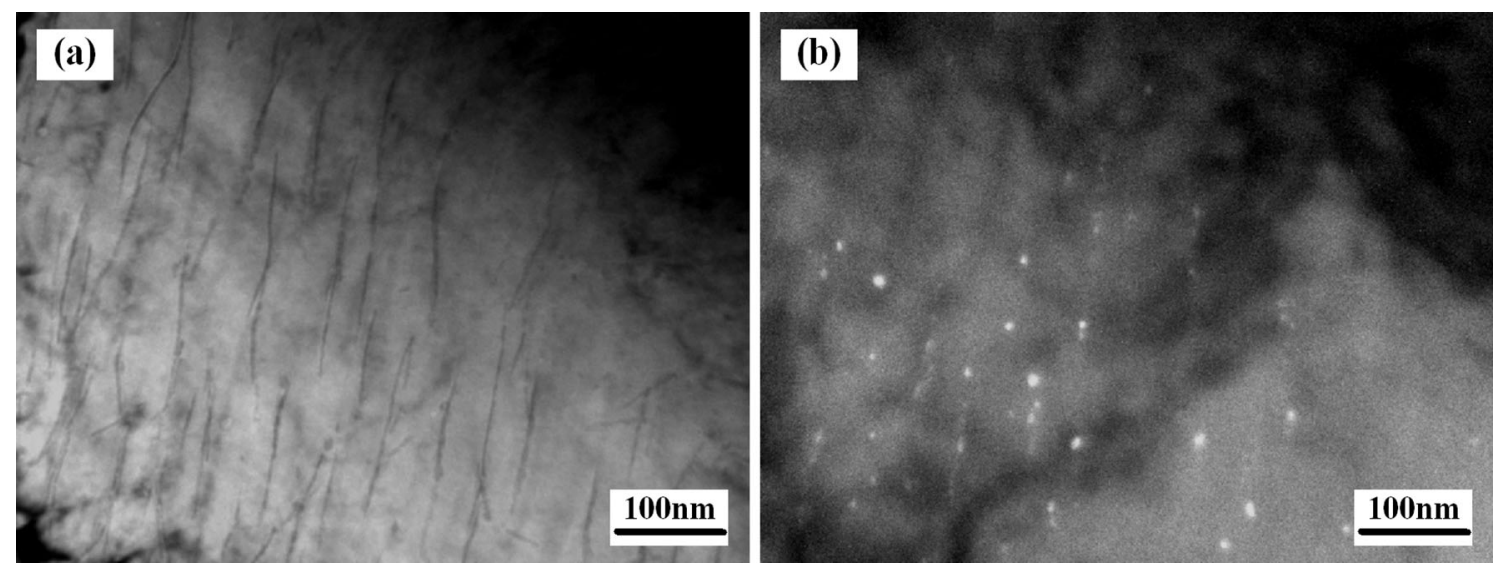

Fig. 12 TEM micrographs of VC precipitates nucleated on dislocations near the ferrite grain boundary after isothermal transformation at $650{ }^{\circ} \mathrm{C}$ for 100 s: a bright-field image; $\mathbf{b}$ dark-field image 
formation process of fibrous precipitates is illustrated in Fig. 11d.

\subsection{Precipitation Sequence of Different Morphologies of VC}

Five morphologies of VC precipitation including precipitation in austenite, planar interphase precipitation, needlelike interphase precipitation, fibrous precipitation and matrix precipitation are found in the V-microalloyed medium-carbon steel. They usually take place in the same ferrite grains, even in the same regions in ferrite. Therefore, it is necessary to ascertain the precipitation sequence between them.

As shown in Fig. 4b, the coarse VC particles firstly nucleate in austenite along the grain boundaries, which form during the cooling process below the austenitization temperature, resulting in a larger size due to the subsequent growth and a precipitate-free zone around the coarse particles.

Khalid and Edmonds [9] developed a model alloy that can preserve the austenite and the transformation interface to room temperature to examine the exact locations of interphase precipitation. It showed the possibility of interphase precipitation occurring ahead of the interface, but it required a long time to be identified. Adequate experimental evidences have proved that the interphase precipitates nucleate at the transformation interface of $\gamma / \alpha$ or $\gamma /$ pearlite. Therefore, the interphase precipitation takes place after the VC precipitation in austenite. Because the needlelike VC particles precipitate at a slowly moving interface, the needlelike interphase precipitation will occur after the planar interphase precipitation. This is also confirmed by the fact that the nucleation site of needlelike precipitates locates inside the pro-eutectoid ferrite as presented in Fig. 3c.

The fibrous VC precipitates and randomly distributed particles were formed in pro-eutectoid ferrite and pearlitic ferrite precipitate after the interphase precipitation. This is also confirmed by the fact that they were observed in the regions where the interphase precipitates are absent, as shown in Figs. 5a and 6b.

The fine particles rarely form around the long and short fibers as presented in Fig. 8a, c, indicating that the fibrous precipitation precedes the matrix precipitation. Because the dislocations are the preferential sites for nucleation, VC precipitates can easily nucleate and grow along the dislocations. The fibrous precipitates consume the carbon and vanadium, leading to the absence of matrix precipitation around.

The precipitation sequence of various morphologies of $\mathrm{VC}$ precipitates is summarized in Table 1.

\section{Conclusions}

1. Linear arrays of fine interphase precipitates, fine needles of interphase precipitates, fibrous precipitates and randomly distributed particles of $\mathrm{VC}$ can precipitate in both pro-eutectoid ferrite and pearlite after the isothermal transformation.

2. The banded interphase precipitates parallel to the $\gamma / \alpha$ interface and the needlelike interphase precipitates perpendicular to the $\gamma / \alpha$ interface exist separately or simultaneously, displaying a single variant of the BN orientation relationship with ferrite.

3. Long fibers and short fibers of VC precipitates nucleate and grow along the dislocations generated during transformation, while the randomly distributed particles as well as the short fibers precipitate almost everywhere displaying multiple variants of the $\mathrm{BN}$ orientation relationship.

4. The precipitation sequence of $\mathrm{VC}$ precipitates is precipitation in austenite, planar interphase precipitation, needlelike interphase precipitation, fibrous precipitation and matrix precipitation.

Acknowledgements The author would like to thank Prof. Y. Todaka from TUT for the discussion in the research and Prof. Z.Q. Hu from IMR for the help in the preparation of this paper.

\section{References}

[1] Y.Q. Wang, S.J. Clark, V. Janik, R.K. Heenan, P.D. Lee, Acta Mater. 145, 84 (2018)

[2] X.L. Li, C.S. Lei, X.T. Deng, Y.M. Li, Y. Tian, Z.D. Wang, G.D. Wang, Acta Metall. Sin. (Engl. Lett.) 30, 1067 (2017)

[3] E. Abbasi, W.M. Rainforth, J. Mater. Sci. Technol. 33, 311 (2017)

[4] K.S. Kim, L.X. Du, C.R. Gao, Acta Metall. Sin. (Engl. Lett.) 28, $692(2015)$

[5] R. Lagneborg, S. Zajac, Metall. Mater. Trans. A 31, 1 (2000)

[6] D.V. Edmonds, J. Iron Steel Inst. 210, 363 (1972)

[7] A. Barbacki, R.W.K. Honeycombe, Metallography 9, 277 (1976)

[8] J.A. Todd, P. Li, Metall. Trans. A 17, 1191 (1986)

[9] F.A. Khalid, D.V. Edmonds, Mater. Sci. Technol. 9, 384 (1993)

[10] R.M. Smith, D.P. Dunne, Mater. Forum 11, 166 (1988)

[11] F.A. Khalid, Mater. Sci. Eng., A 325, 281 (2002)

[12] S. Gunduz, A. Capar, J. Mater. Sci. 41, 561 (2006)

[13] H.H. Kuo, M. Umemoto, K. Sugita, G. Miyamoto, T. Furuhara, ISIJ Int. 52, 669 (2012)

[14] H.W. Yen, C.Y. Huang, J.R. Yang, Scripta Mater. 61, 616 (2009)

[15] G. Fourlaris, A.J. Baker, G.D. Papadimitriou, Acta Metall. Mater. A 43, 3733 (1995)

[16] R. Okamoto, J. Ågren, Acta Mater. 58, 4791 (2010)

[17] R.A. Ricks, P.R. Howell, Metal. Sci. 16, 317 (1982)

[18] R.A. Ricks, P.R. Howell, Acta Metall. 31, 853 (1983)

[19] T. Obara, G.J. Shiflet, H.I. Aaronson, Metall. Trans. A 14, 1159 (1983)

[20] P. Li, J.A. Todd, Metall. Trans. A 19, 2139 (1988) 